\title{
PROBABILITY OF SHOCK IN THE PRESENCE AND ABSENCE OF CS IN FEAR CONDITIONING ${ }^{1}$
}

\author{
ROBERT A. RESCORLA \\ Yale University
}

\begin{abstract}
2 experiments indicate that CS-US contingency is an important determinant of fear conditioning and that presentation of US in the absence of CS interferes with fear conditioning. In Experiment 1, equal probability of a shock US in the presence and absence of a tone CS produced no CER suppression to CS; the same probability of US given only during CS produced substantial conditioning. In Experiment 2, which explored 4 different probabilities of US in the presence and absence of CS, amount of conditioning was higher the greater the probability of US during CS and was lower the greater the probability of US in the absence of CS; when the 2 probabilities were equal, no conditioning resulted.
\end{abstract}

Two conceptions of Pavlovian conditioning have been distinguished by Rescorla (1967). The first, and more traditional, notion emphasizes the role of the number of pairings of CS and US in the formation of a $\mathrm{CR}$. The second notion suggests that it is the contingency between CS and US which is important. The notion of contingency differs from that of pairing in that it includes not only what events are paired but also what events are not paired. As used here, contingency refers to the relative probability of occurrence of US in the presence of CS as contrasted with its probability in the absence of CS. The contingency notion suggests that, in fact, conditioning only occurs when these probabilities differ; when the probability of US is higher during CS than at other times, excitatory conditioning occurs; when the probability is lower, inhibitory conditioning results. Notice that the probability of a US can be the same in the absence and presence of CS and yet there can be a fair number of CS-US pairings. It is this that makes it possible to assess the relative importance of pairing and contingency in the development of a CR.

Several experiments have pointed to the usefulness of the contingency notion. Rescorla (1966) reported a Pavlovian

\footnotetext{
${ }^{1}$ This research was supported by Grants $\mathrm{MH}-$
} 13415-01 from the National Institute of Mental Health and GB-6493 from the National Science Foundation, as well as by funds from Yale University. fear conditioning experiment with dogs, using CS-induced changes in avoidance rate as an index of fear. Three groups were run: (a) a random group in which the probability of US was the same in the presence and absence of CS; (b) an excitatory conditioning group in which the probability of US during CS was the same as in the random group but for which US never occurred in the absence of CS; and (c) an inhibitory group in which the probability of US in the absence of CS was the same as in the random group but for which US never occurred during CS. Later presentation of these stimuli in the course of free-operant avoidance behavior indicated that the second group showed fear conditioning to CS, the third group showed inhibition of fear, and the first group showed no evidence of any conditioning. This result occurred despite the fact that the first and second groups had the same number of CS-US pairings and differed only in the degree to which the US was uniquely paired with the CS. The experiments reported here extend this result.

\section{EXPERIMENT 1}

In Experiment 1, the Rescorla (1966) finding is essentially replicated with a different organism and a further control procedure is run.

\section{Method}

Subjects. Twenty-four male Sprague-Dawley rats, about 150 days old at the start of the experi- 
ment, were maintained in individual cages at $80 \%$ of their normal body weight.

Apparatus. Each of four identical $9 \times 8 \times 8$-in. Skinner boxes had a recessed food magazine in the center of the end wall and a retractable lever to the left of the magazine. During Pavlovian conditioning sessions, aluminum blanks covered the magazine and bar apertures. The floor of the chamber was composed of $3 / 16$-in. stainless-steel rods spaced $3 / 4$ in. apart and could be electrified through a relay-sequence scrambler (Hoffman \& Fleshler, 1962) from a high-voltage high-resistance shock source. The two end walls of the chambers were aluminum; the side walls and top were clear Plexiglas. Mounted on the ceiling of the sound and light resistant ice chest, which enclosed each

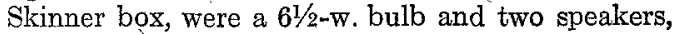
permitting the presentation of a constant white masking noise and of a $720-c p s$ tone CS. Experimental events were controlled and recorded automatically by relay equipment located in an adjoining room.

Procedure. Each $S$ was maintained at $80 \%$ body weight for 1 wh. prior to the first experimental session during which $S$ was magazine trained automatically with food pellets delivered on a 1-min. VI schedule. In addition, each bar press yielded a food pellet. This session continued until $S$ had emitted about 60 bar presses; shaping was used if necessary. Starting with the second experimental day, all sessions were $2 \mathrm{hr}$. long and $S$ was placed on a VI schedule of reinforcement. For the first $30 \mathrm{~min}$. of this session the schedule was I-min. VI; thereafter it was 2-min. VI. After 5 days of VI training, the bars were removed from the Skinner boxes and five daily sessions of Pavlovian fear

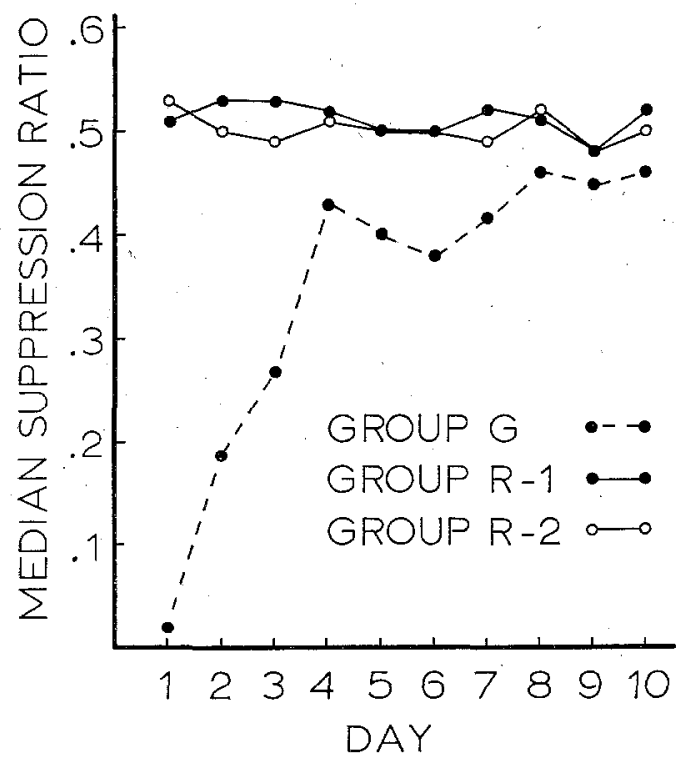

FIa. 1. Median suppression ratio for each group over the ten test sessions of Experiment 1 . conditioning were administered. For eight $S$ s in Group R-1 (random), 12 2-min. tone CSs were given in each session with a mean intertone interval of $8 \mathrm{~min}$. with 12.5 -sec. .9-ma. electric shocks programmed randomly, throughout the session; shocks were programmed independently of the tones in such a way that shock was equiprobable at any time within the session. The eight $S$ s in Group G (gated) received a treatment identical to that of Group R-1 except that all shocks which would have occurred in the absence of the tone CS were simply omitted. This means that on the average $S$ s in Group $G$ received the same number of shocks during CS as $S \mathrm{~s}$ in Group $\mathrm{R}-1$, but $S \mathrm{~s}$ in Group G received no shocks in the absence of $\mathrm{CS}$ and therefore fewer total shocks. The eight $S$ s in Group R-2 received a treatment identical to those of Group R-1 except that the average number of shocks they received (2.4 per session) was the same as that for Group $G$. These shocks were programmed randomly throughout the session, independently of the tones.

Following Pavlovian conditioning; two 2-hr. VI sessions were given to assure a stable bar-pressing rate, followed by ten test sessions. During each 2 -hr. test session 2-min. VI reinforcement remained in effect for bar pressing; superimposed upon this performance were four 2 -min. presentations of the tone $\mathrm{CS}$, with a mean intertrial interval of $30 \mathrm{~min}$. No shocks were administered during any of these. sessions.

In order to attenuate the effects of individual differences in general rate of responding, results are plotted in terms of a suppression ratio of the form $A /(A+B)$ where $A$ is the rate of responding in $\mathrm{CS}$ and $\mathbf{B}$ is the rate of responding in a comparable period prior to CS onset. Thus a suppression ratio of 0 indicates no responding during CS while one of .5 indicates similar rates of responding during CS and the pre-CS period. Rates of responding in the pre-CS period were not reliably different for the three groups.

\section{Results}

It is clear from Figure 1 that the presentation of $\mathrm{CS}$ had little effect upon Groups R-1 and R-2. Throughout testing they responded similarly in the pre-CS and CS periods. In contrast, however, the CS produced a sharp reduction in response rate in Group G. With repeated presentation of CS in the absence of all shocks, the suppression in Group $G$ extinguished almost to the level of the two random groups. An analysis of variance performed on the suppression scores for the three groups over the ten extinction sessions showed a significant difference among groups $(F=15.38, d f=2 / 21, p<.01)$ and an effect of extinction days $(F=8.80$, 
$d f=18 / 189, p<.01$ ). Individual comparisons indicated that these effects were due to the difference between Group G and the other two groups.

Response suppression was not uniform throughout CS for Group G; suppression was maximum at CS onset and became attenuated as CS continued. Figure 2 shows the distribution of suppression in four 30-sec. segments of CS over the first 5 extinction days. A two-way analysis of variance confirmed the observation that suppression was greatest at CS onset ( $F=$ $4.69, d f=4 / 28, p<.01$ ) as well as the obvious extinction of suppression over days. In addition, the Extinction Days $\times$ Periods during CS interaction was reliable $(F=$ $2.16, d f=12 / 84, p<.05$ ), indicating that extinction of suppression occurred first for the later periods during CS.

\section{Discussion}

The results of this experiment confirm the earlier findings of Rescorla (1966). Only when the probability of shock was higher during CS than at other times did conditioning occur. Groups with either the same number of CS-US pairings or the same number of USs, but lacking an increased probability of US during CS, failed to condition. These results add considerable weight to the contention that in Pavlovian fear conditioning the contingency of US upon CS is of primary importance.

\section{EXPERIMENT 2}

Previous experiments have compared only a few levels of the contingency of US upon CS. In Experiment 1, a high degree of dependence (in Group G) was compared with complete independence (Groups R-1 and R-2). The present experiment explores intermediate contingency relations in an attempt to assess the contingency notion over a wider range of experimental conditions.

\section{Method}

Subjects. Eighty male Sprague-Dawley rats, about 100 days old at the start of the experiment, were maintained in individual cages at $80 \%$ of their normal body weight.
Apparatus. The apparatus was that of Experiment 1 , except that different chambers were used during the conditioning phase. These chambers were identical to the Skinner boxes used in VI training, except that both end walls were plain aluminum.

Procedure. The Ss were bar-press trained in the manner described in Experiment 1. After five 2-hr. sessions of VI bar pressing, five daily $2 \mathrm{hr}$. Pavlovian conditioning sessions were administered. The $S_{\mathrm{s}}$ were divided into ten groups, each receiving a different probability of shock during the presence and absence of CS. For all groups, 12 2-min. 720-cps tones were administered with a mean intertone interval of $8 \mathrm{~min}$. The different probabilities of shock per 2-min. interval were $.4-.4, .2-.2, .1-.1,0-0, .4-.2, .4-.1, .4-0, .2-.1, .2-0$, $.1-0$, where the first number designates the probability of shock in CS and the second the probability of shock in the absence of CS. Shocks of .5sec. duration at $.9 \mathrm{ma}$. were randomly distributed throughout the session in such a way that they had a fixed probability in each second of the session; the occurrence of CS changed that probability value appropriately. Only the expected probability of USs were explicitly programmed; the actual sequence of USs was generated separately for each $S$ during each conditioning session.

After five conditioning sessions, two 2-hr. VI sessions were given to assure a stable bar-pressing rate during the subsequent 6 days of test sessions; during each test session VI-2 reinforcement remained in effect and four 2-min. CSs were presented with a $30-\mathrm{min}$. mean intertone interval. No shocks were administered during any of these sessions.

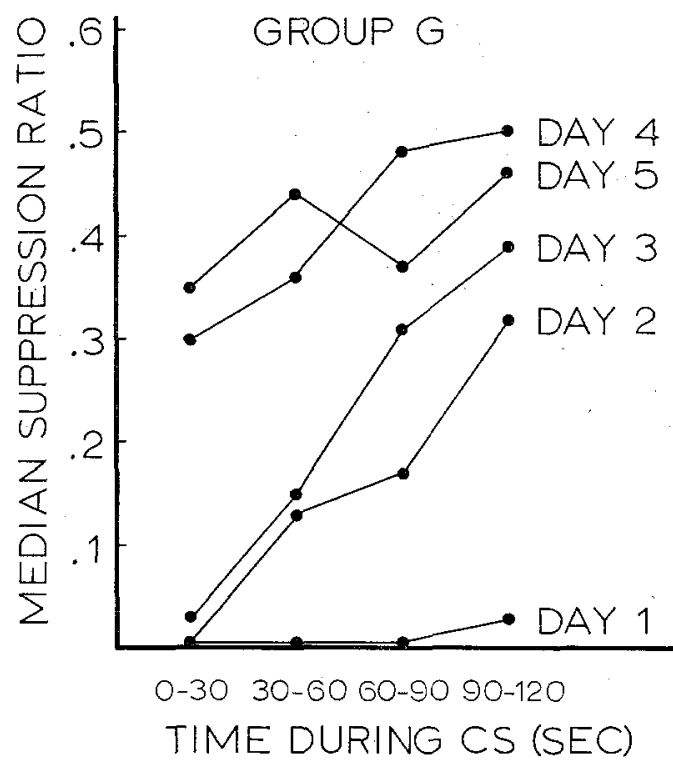

FIG. 2. Distribution of suppression during CS for Group $G$ on each of the first 5 test days. 


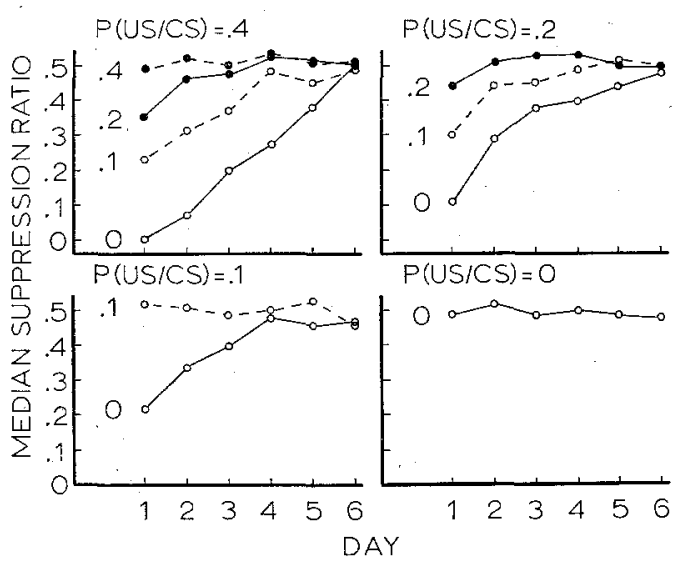

FIg. 3. Median suppression ratio for each group over the six test sessions of Experiment 2. (Within each panel, all groups have the same probability of US during CS; the parameter in each panel is the probability of US in the absence of CS.)

\section{Results}

Despite the fact that different groups received different total numbers of shocks, there were no reliable differences among groups in responding in the absence of CS on either VI training or tests days that followed conditioning.

Figure 3 shows the suppression ratios during the six extinction test sessions. It is clear that within each panel, the groups order themselves according to the probability of shock in the absence of CS, with the lowest probability producing the most suppression. When the probability of shock in the presence and absence of CS is the same there is little or no suppression. Comparing panels, it is also clear that with a fixed probability of shock in the absence of CS, increasing the probability of shock in its presence produced increasing suppression. An overall analysis of variance indicated that these observations were reliable. There was a significant effect of groups $(F=11.69$, $d f=9 / 70, p<.01)$, of extinction days $(F=79.84, d f=5 / 320, p<.01)$, and a Groups $\times$ Extinction Days interaction $(F=10.58, d f=45 / 320, p<.01)$. Individual comparisons on the first test day, using Duncan's multiple range test, indicated that within each panel all pairwise differences were reliable. Across panels, the four groups with equal probability of shock during and in the absence of CS did not differ from each other.

As in Experiment 1, suppression was not uniform through CS. For those groups showing response decrement during $\mathrm{CS}$, the decrement was maximal at CS onset and became less as CS continued. Groups which failed to show overall suppression responded relatively uniformly throughout CS.

\section{Discussion}

The results of this experiment can be succinctly described by three statements. (a) Independent of the overall probability of shock, there was little or no suppression if shock came with equal probability in the presence and absence of CS. (b) With a fixed probability of shock during CS, increasing the probability in the absence of CS attenuated suppression. (c) With a fixed probability of shock in the absence of CS, increasing the probability of shock in the presence of CS enhanced suppression.

Statement $(c)$ agrees with the traditional notion that it is the number of simultaneous occurrences of CS and US that is important in conditioning. However, the amount of suppression obtained cannot be entirely accounted for in terms of either the total number of US eventsor the number of USs during CS. For instance, Group .1-0 received far fewer shocks during CS than did Group .4-.4, yet showed considerably greater suppression.

Any account of the present data must include the disruptive effects upon conditioning of shocks occurring in the absence of CS. One way of viewing this effect has been suggested by Rescorla and LoLordo (1965). They found that presenting USs exclusively in the absence of CS led to the establishment of that CS as a conditioned inhibitor of the CR. They suggested that such a conditioning procedure, presenting USs in the absence of CS, leads to the development of Pavlovian inhibition in response to CS. One can, then, view the present results as the product of two separate acquisition processes: (a) the development of Pavlovian conditioned 
excitation as a result of CSs and USs occurring together, and $(b)$ the development of Pavlovian conditioned inhibition as a result of USs occurring in the absence of CS. It is perhaps fortuitous that these two processes are balanced when there is equal probability of US in the presence and absence of CS.

Alternatively, one might view these results in terms of attention to CS. It might be argued that conditioning occurs, as has traditionally been thought, whenever CS and US occur together; however, presentation of US at other times leads the organism to attend to a variety of stimuli other than CS. Thus the disruption of suppression as a result of USs occurring in the absence of CS represents not a failure of Pavlovian conditioning but a failure of $S$ to attend to the CS during testing. Our task then becomes one of specifying the variables controlling this attention. Although such an account is plausible for the present data, it fails to explain the active inhibition of fear found by Rescorla and LoLordo (1965), Rescorla (1966), and Hammond (1967).

One unexpected finding of these experiments was that suppression was not uniform during CS. This seems surprising on the contingency view which emphasizes the instantaneous probability of US, for that probability is constant throughout CS. There are several possibilities for understanding this distribution of suppression during CS in terms consistent with the contingency viewpoint. One possibility is that the reduced suppression later in the $\mathrm{CS}$ is an artifact of the measuring technique. A VI schedule of reinforcement is such that the longer $S$ has refrained from pressing, the higher the probability that its next press will be reinforced. Thus the longer $S$ suppresses, the more "pressure" the base-line operant schedule places on it to respond. Fear conditioning may be constant throughout, only the tendency to press, which fear counteracts, may be changing. A second possibility is simply that the onset of CS is a more discriminable stimulus than its continued presence and thus shows superior conditioning even with equal probabilities of US.

\section{REFERENCES}

Hammond, L. O. A traditional demonstration of the active properties of Pavlovian inhibition using differential CER. Psychon. Sci., 1967, 9, $65-66$.

Hoffman, H. S., \& Fleshler, M. A relay sequencing device for scrambling grid shock. $J$. exp. Anal. Behav., 1962, 5, 329-330.

Rescorla, R. A. Predictability and number of pairings in Pavlovian fear conditioning. Psychon. Sci., 1966, 4, 383-384.

Rescorla, R. A. Pavlovian conditioning and its proper control procedures. Psychol. Rev., 1967, 74, 71-80.

Rescorla, R. A., \& LoLoRdo, V. M. Inhibition of avoidance behavior. J. comp. physiol. Psychol., $1965,59,406-412$.

(Received August 17, 1967) 